\title{
Waveguide Dispersion Tailoring by Using Embedded Impedance Surfaces
}

\author{
Yijing He, ${ }^{1}$ Yue Li, ${ }^{1,}{ }^{*}$ Liang Zhu, ${ }^{2}$ Hakan Bagci, ${ }^{3}$ Danilo Erricolo, ${ }^{2}$ and Pai-Yen Chen ${ }^{2}$ \\ ${ }^{1}$ Department of Electronic Engineering, Tsinghua University, Beijing 100084, China \\ ${ }^{2}$ Department of Electrical and Computer Engineering, University of Illinois, Chicago, Illinois 60607, USA \\ ${ }^{3}$ Division of Computer, Electrical, and Mathematical Sciences and Engineering, King Abdullah University of \\ Science and Technology (KAUST), Thuwal 23955-6900, Saudi Arabia
}

(Received 2 May 2018; revised manuscript received 4 October 2018; published 11 December 2018)

\begin{abstract}
The capability to tailor the dispersion and the cut-off frequency of waveguides is of importance, as these essential parameters govern the operating frequency range and the waveguide dimension. Here, we propose the concept of substrate-integrated impedance surface (SIIS) that enables arbitrary control of propagation characteristics of closed-shape waveguides. Specifically, we develop a theoretical framework for the simplest form of SIIS constituted by a one-dimensional array of blind vias, which is equivalent to a homogenized surface capacitance embedded in the waveguide. We theoretically and experimentally demonstrate that loading a substrate-integrated waveguide (SIW) with a capacitive SIIS can effectively reduce its cut-off frequency, regardless of the transverse dimension of the SIW. In addition, a SIIS-loaded SIW exhibits several intriguing phenomena, such as the slow-wave guiding properties and the local field concentration. This SIIS-loading technique may open up new possibilities for miniaturization of various waveguide-based components and for enhancement of their uses in microwave sensing and nonlinear functions.
\end{abstract}

DOI: 10.1103/PhysRevApplied.10.064024

\section{INTRODUCTION}

Waveguides have had a major role in the development of modern communication and sensing systems, as they have the advantages of high power-handling capability and low transmission loss. Potential applications include the high-power microwave and millimeter-wave circuits, radar and satellite communication systems, waveguidebased sensors, and photonic integrated circuits, to name a few [1-3]. Moreover, except for being used as an interconnect for signal transmission, rectangular waveguides can be extensively used to implement electromagnetic components, such as antennas (if suitably opened or slotted) $[4,5]$, filters $[6,7]$, resonators $[8,9]$, couplers $[10,11]$, and phase shifters $[12,13]$. The cut-off frequency and the dispersion curve are major specifications for a waveguide. Below the cut-off frequency, a waveguide cannot transmit electromagnetic waves, analogous to a high-pass energy conduit. In this context, Engheta and several groups have recently reported that a rectangular waveguide operated slightly above the cut-off frequency can be treated as an effective zero-index medium, because the guided wave exhibits a nearly infinite phase velocity (fast-wave propagation), along with almost uniform and intense electric fields inside the waveguide. Such properties enable many

\footnotetext{
*1yee@tsinghua.edu.cn
}

new applications, such as supercoupling and tunneling [14-18], enhanced nonlinear electromagnetic responses [19-21], and Purcell enhancement of molecular emissions [22]. Recently, substrate-integrated waveguides (SIWs) [Fig. 1(a)] have received great popularity in microwave and millimeter-wave circuits, due to their unique advantages of high power-carrying capacity, high quality factor ( $Q$ factor), and good compatibility with high-yield printed circuit board (PCB) $[23,24]$ and CMOS manufacturing processes $[25,26]$. Basically, a SIW offers the benefits of a bulky rectangular waveguide, but with a low-profile and light-weight planar structure.

In this paper, we propose the concept of substrateintegrated impedance surface (SIIS) or "implanted metasurface" to tailor the cut-off frequency, dispersion, physical size, and field distributions of a SIW or any closed waveguide. Figure 1 presents the general concept of the proposed SIIS. For instance, a SIIS could be formed by a one-dimensional (1D) blind-via array [Fig. 1(b)], which, together with its mirror image inside the waveguide, effectively makes an impedance surface with surface impedance $Z_{s}=i /\left(\omega C_{s}\right)$, where $C_{s}$ is the surface capacitance. Such an impedance sheet embedded in a waveguide is somehow similar to a two-dimensional (2D) metasurface or frequency selective surface (FSS), whose equivalent surface impedance is tailored by geometry and a period of repeated inclusions (electromagnetic scatters). We note 


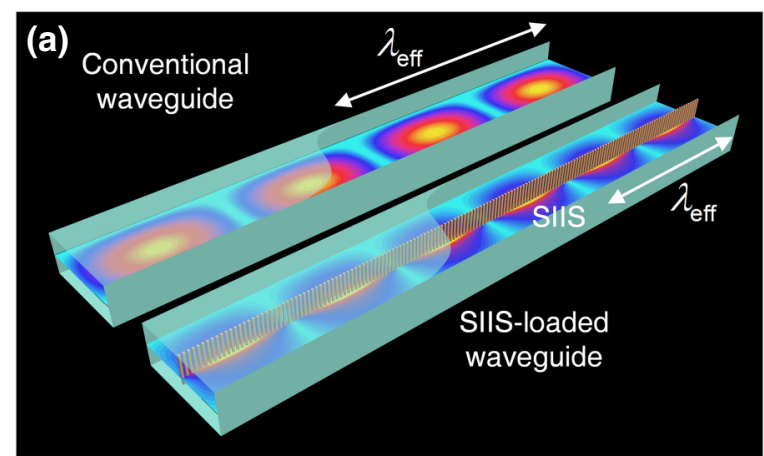

(b)

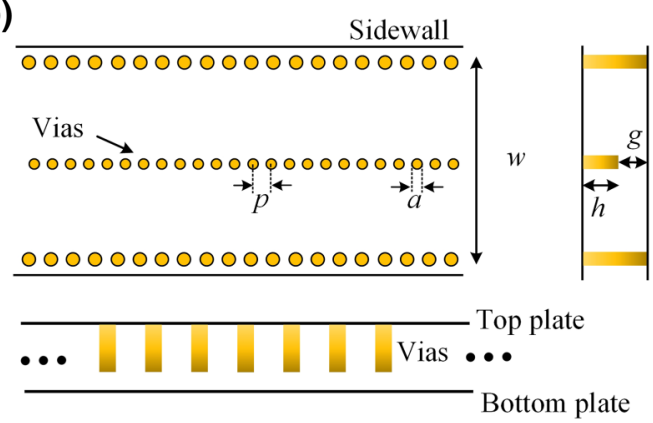

(c)
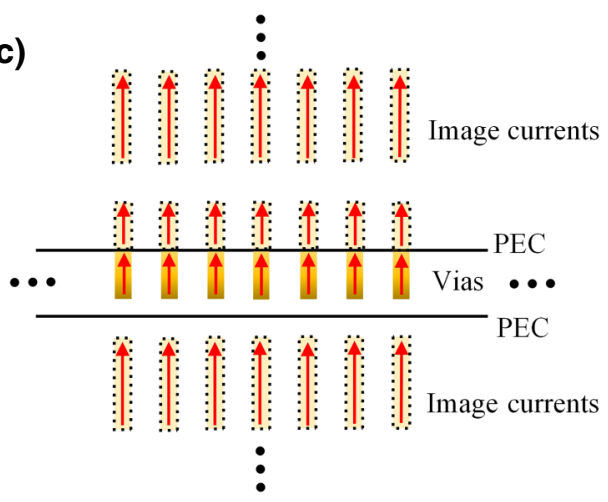

FIG. 1. The general concept and structure of SIIS. (a) Schematics of the standard waveguide (top) and the SIIS-loaded waveguide (bottom); $\lambda_{\text {eff }}$ represents the effective wavelength in the waveguide channel. (b) The top, cross-section, and longitudinal views of a SIIS-loaded SIW. (c) Equivalence of SIIS to a 2D impedance surface, formed by currents induced on the blind-vias array and their images.

that a compact SIIS, when loaded into a SIW using a compatible manufacturing process, could tailor the dispersion characteristics, cutoff, and modal profile, and field distributions of a SIW, thus creating a number of new applications. One potential application is to tailor the epsilon-near-zero (ENZ) responses of a waveguide with the working frequency tunable over a wide range and independent of the waveguide's physical dimension. Thanks to the reduced cutoff and the reduced effective wavelength in the SIIS-loaded waveguide, related to the slow-wave propagation, the proposed technique also enables extreme miniaturization of various waveguide-based components (e.g., resonators, filters, couplers, hybridizers, and phase shifters). In addition, the large field enhancement in the proximity of the reactive SIIS may be used to make ultrasensitive microwave sensors and efficient nonlinear components (e.g., an active waveguide loaded with high-power electronic devices). In this work, we will derive the explicit formula to describe the surface impedance $\left(Z_{s}\right)$ for the SIIS in Fig. 1, with a specially designed geometry. Once the surface impedance of SIIS is determined, the complex propagation constant and dispersion characteristics of a SIIS-loaded waveguide can be calculated by solving the transcendental equation. To validate the proposed concept, we will conduct numerical simulations and experimental characterizations for different on-board SIIS-loaded SIWs.

\section{THEORETICAL ANALYSIS FOR THE SUBSTRATE-INTEGRATED IMPEDANCE SURFACE (SIIS)}

Here, we consider a capacitive SIIS in Fig. 1(b), consisting of a 1D array of blind vias with subwavelength diameter $a$ and period $p$, which is separated from the bottom ground plane by a small gap $g$. At microwave frequencies, a single blind via (through-hole via) with height $h$ placed between the top and bottom metal plates of a SIW can be equivalent to one column of discrete (continuous) wire array, as shown in Fig. 1(c). As a result, a physical wire and its imaged counterpart mirrored by the metal layer [i.e., perfect electric conductor or PEC with $(\varepsilon, \mu)=$ $\left.\left(-\infty, \mu_{0}\right)\right]$ may effectively form a shunt impedance surface implanted inside a SIW. In this scenario, the tangential magnetic fields on the two sides of a SIIS are related to the tangential electric fields by the surface impedance $Z_{s}: E_{x+}=E_{x-}=Z_{s}\left[\hat{z} \times\left(H_{y+}-H_{y-}\right)\right]$. As we will derive in the following, the blind-vias array can be treated as a capacitive impedance surface with a homogeneous $Z_{s}$ along the longitudinal direction. Likewise, the subwavelength through-hole vias can form the two PEC sides of a SIW. In fact, the through-hole vias array is equivalent to an inductive surface impedance $Z_{s}=-i \omega L_{s}$, where $L_{s}$ is the surface inductance. The through-hole via array can be treated as a PEC sheet if $L_{s}$ is small or the operating frequency is low (i.e., short circuit) [23]. The dispersion equation of a SIIS-loaded SIW can be derived using the transverse resonance method [27], and, for the dominant $\mathrm{TE}_{10}$ mode, it is given by

$$
\frac{-i \omega \mu_{0}}{2 \sqrt{k^{2}-\beta^{2}}} \tan \left[\sqrt{k^{2}-\beta^{2}} \frac{w}{2}\right]+Z_{s}(\omega)=0,
$$

where $k=\omega \sqrt{\varepsilon \mu_{0}}, \omega$ is the angular frequency, $w$ is the width of SIW, $\varepsilon$ is the permittivity of the dielectric medium inside the SIW; the $\exp (-i \omega t)$ time convention is adopted throughout this paper. It is rather illustrative to study an extremely miniaturized SIW. It can be readily understood from Eq. (1) that in the quasi-static limit $(k w \ll 1)$, 
a capacitive surface impedance, $Z_{s}\left(\omega_{0}\right)=i \omega_{0} \mu_{0} w / 4$, is required to excite a propagating mode at the frequency $\omega_{0}$. In essence, there is no limit on the cut-off frequency, provided that the required $Z_{s}$ can be achieved at the working frequency.

When an implanted impedance surface (or metasurface) formed by discrete wires is excited by an external electric field $\mathbf{E}_{\text {ext }}$ with the TE polarization, the collective behavior of the induced electric dipole moments will result in a homogenous, thin sheet of surface current. The total averaged polarization related to the local field $\mathbf{E}_{\text {loc }}=E_{\mathrm{loc}} \hat{\boldsymbol{x}}$ is responsible to the component $\alpha_{x x}$ of the electric polarizability tensor $\overline{\bar{\alpha}}$. Considering the full coupling among the whole array of interacting electric dipoles (subwavelength cutwires [Fig. 1(a)]), the dipole moment induced on the nanoparticle is given by [28-31]

$$
\begin{array}{r}
\mathbf{P}_{N x N y}=\alpha_{x x} \mathbf{E}_{\mathrm{loc}}=\alpha_{x x}\left[\mathbf{E}_{\mathrm{ext}}+\sum_{\left(N_{x}, N_{y}\right) \neq\left(N_{x}^{\prime}, N_{y}^{\prime}\right)}\right. \\
\left.\mathbf{G}\left(\mathbf{r}_{N_{x} N_{y}}-\mathbf{r}_{N_{x}^{\prime}, N_{y}^{\prime}}\right) \cdot \mathbf{P}_{N_{x}^{\prime}, N_{y}^{\prime}}\right],
\end{array}
$$

where $N_{x}$ and $N_{y}$ are positive or negative integers, which relate the position of local dipoles and periods $d_{x}$ (here, $d_{x}=p$ ) and $d_{y}$ (here, $d_{y}=2 h+2 g$ ) by $x=N_{x} d_{x}$ and $y=N_{y} d_{y}, \mathbf{r}_{N_{x} N_{y}}$ is the position of the $\mathbf{P}_{N x N y}$ dipole, and $\mathbf{G}\left(\mathbf{r}_{N_{x} N_{y}}, \mathbf{r}_{N_{x}^{\prime} N_{y}^{\prime}}\right)=\left(\nabla \nabla+k^{2} \overline{\overline{\mathbf{I}}}\right)\left[e^{-j k\left|\mathbf{r}_{N_{x} N_{y}}-\mathbf{r}_{N_{x}^{\prime} N_{y}^{\prime}}\right|} /\left(4 \pi \mid \mathbf{r}_{N_{x} N_{y}}\right.\right.$ $\left.\left.-\mathbf{r}_{N_{x}^{\prime} N_{y}^{\prime}} \mid\right)\right]$ is the dyadic Green's function in free space. After some algebraic manipulations, we may write Eq. (2) as

$$
\mathbf{P}_{00}=P_{00} \hat{x}=\frac{\mathbf{E}_{\text {ext }}}{\alpha_{x x}^{-1}-C_{\text {int }}},
$$

where $C_{\text {int }}=\sum_{\left(N_{x}, N_{y}\right) \neq(0,0)} \mathbf{G}\left(\mathbf{r}_{N_{x} N_{y}}, \mathbf{r}_{N_{x}^{\prime} N_{y}^{\prime}}\right) \cdot \hat{\mathbf{x}} \cdot \hat{\mathbf{x}}$ is the interaction constant that relates the fields induced by the infinite array of electric dipoles around the unit cell under consideration to the local field at the origin. The averaged surface current density on the SIIS can be expressed as

$$
\mathbf{J}_{s}=J_{s} \hat{\boldsymbol{x}}=\frac{-i \omega \mathbf{P}_{00}}{d_{x} d_{y}}=\frac{-i \omega E_{\mathrm{ext}}}{d_{x} d_{y}\left(\alpha_{x x}^{-1}-C_{\mathrm{int}}\right)} \hat{\mathbf{x}} .
$$

This is an accurate description of the array's electromagnetic properties, as long as the periods are small enough to ensure that only one Floquet harmonic could exist. The average sheet impedance of the SIIS, as the ratio of the local electric field to the surface current density, can be expressed as

$$
Z_{s}=\frac{E_{\mathrm{ext}}}{J_{s}}-\frac{\eta}{2}=-\frac{d_{x} d_{y}}{i \omega}\left(\alpha_{x x}^{-1}-C_{\mathrm{int}}\right)-\frac{\eta}{2},
$$

where $\eta$ is the intrinsic impedance of the host medium. In the lossless scenario, which is approximately valid at low frequencies, Eq. (5) may be further manipulated by considering the physical requirements on the imaginary parts of $\alpha_{x x}^{-1}$ and $C_{\text {int }}$, associated with the power balance [32]

$$
\operatorname{Im}\left[\alpha_{x x}^{-1}\right]=-\frac{k^{3}}{6 \pi \varepsilon} \quad \text { and } \quad \operatorname{Im}\left[C_{\text {int }}\right]=-\frac{k^{3}}{6 \pi \varepsilon}+\frac{\eta \omega}{2 d_{x} d_{y}} .
$$

From Eqs. (5) and (6), the surface impedance $Z_{s}$ becomes

$$
Z_{s}=-\frac{d_{x} d_{y}}{i \omega}\left[\operatorname{Re}\left(\alpha_{x x}^{-1}\right)-\operatorname{Re}\left(C_{\mathrm{int}}\right)\right] .
$$

For a single thin and short conducting wire, the real part polarizability can be explicitly written as [32]

$$
\operatorname{Re}\left(\alpha_{x x}\right)=\frac{4 \varepsilon \pi h^{3}}{3[\ln (2 h / a)-1]},
$$

where $h$ and $a$ are the length and radius of the wire. In addition, in the quasi-static limit, the real part of interaction constant is approximately given by [31]

$$
\operatorname{Re}\left(C_{\mathrm{int}}\right) \approx \frac{c}{\varepsilon\left(d_{x} d_{y}\right)^{3 / 2}},
$$

where $c$ is an empirical fitting parameter. From Eqs. (7)-(9), the surface impedance of the SIIS can be expressed as

$$
\begin{aligned}
Z_{s} & =-\frac{1}{i \omega C_{s}} \quad \text { and } \\
C_{s} & =\frac{\left[\varepsilon /\left(d_{x} d_{y}\right)\right]}{\left(\{3[\ln (2 h / a)-1]\} / 4 \pi h^{3}\right)-\left[c /\left(d_{x} d_{y}\right)^{3 / 2}\right]}
\end{aligned}
$$

\section{NUMERICAL AND EXPERIMENTAL DEMONSTRATIONS}

In this section, we theoretically and experimentally demonstrate the capability of a SIIS to engineer the properties of a SIW. As can be understood from Eq. (10), the SIIS in Fig. 1(c) exhibits a surface capacitance that is a function of the gap $g$ and the period $p$ of blind vias. Additionally, $d_{x}=p, d_{y}=2 h+2 g$, and $h$ is the height of the blind vias. Figures 2(a) and 2(b) report the surface capacitance $C_{s}$ for such a SIIS with different geometric parameters, which are calculated using Eq. (10) with the empirical parameter $c=0.13$. From Figs. 2(a) and 2(b), we find that $C_{s}$ is a strong function of geometric parameters, and it increases upon reducing the gap size $(g)$ or period $(p)$. By varying the geometry and the array period of blind vias, the surface reactance of this SIIS can be tuned over a wide range, thus offering flexibility in tailoring the dispersion of the SIW.

Without loss of generality, we consider a lossless, air-filled SIW with width $w=25 \mathrm{~mm}$ and height $h_{0}=h+g=3 \mathrm{~mm}$, where $h$ is the height of the blind vias 
(a)
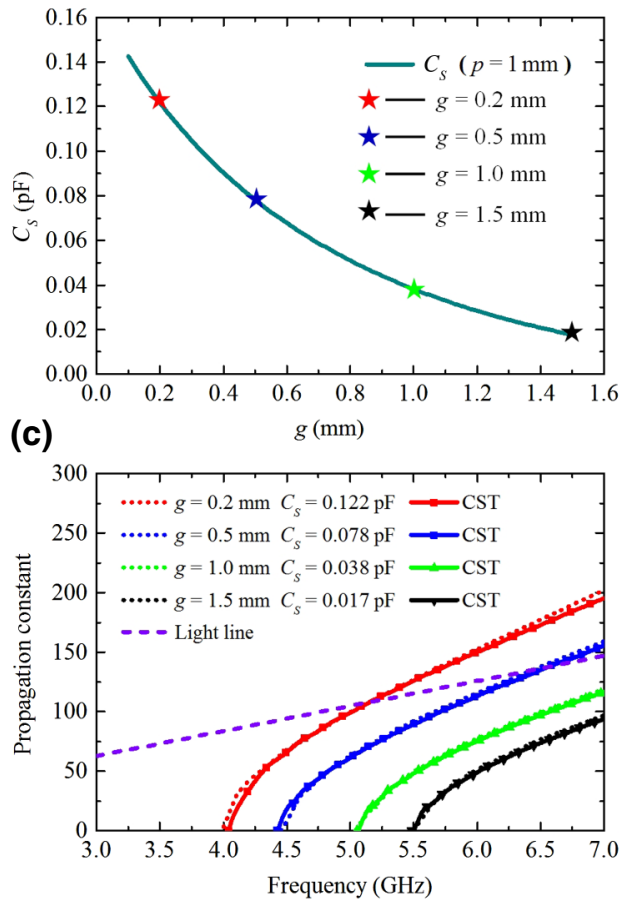

(b)
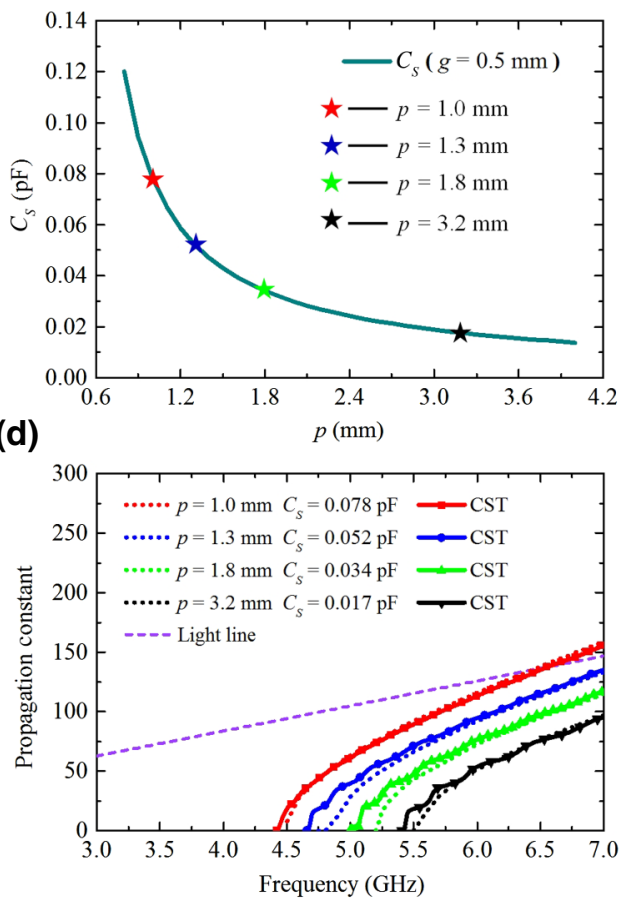

FIG. 2. Theoretical surface capacitance of the SIIS shown in Fig. 1(b) and the comparison of the analytical and numerical dispersion curves of the SIIS-loaded SIW: (a) Theoretical surface capacitance of the SIIS vs the gap $g(p=1 \mathrm{~mm})$. (b) Theoretical surface capacitance of the SIIS vs the period $p(g=0.5 \mathrm{~mm})$. Propagation constant vs frequency for a SIIS-loaded SIW with (c) $p=1 \mathrm{~mm}$ and a variable $g$, and (d) $g=0.5 \mathrm{~mm}$ and a variable $p$; here, the dashed and solid lines are obtained from the analytical model [Eqs. (1) and (10)] and numerical simulation, respectively. and $g$ is the gap size; all blind vias have the same diameter $a=0.6 \mathrm{~mm}$. This SIW exhibits a cut-off frequency of $6 \mathrm{GHz}$. We will show below that the dispersion and the cut-off frequency of a SIW can be tuned by the surface reactance of loaded SIIS, instead of the SIW's transverse dimension. We conduct full-wave numerical simulations to calculate dispersion curves of the SIIS-loaded SIWs in Fig. 1. Our numerical simulations are based on the frequency-domain finite-element method [33], and realistic models are used to describe material dispersions and losses of metals and dielectrics. Figure 2(c) compares analytical (dashed lines) and numerical (solid lines) results for the frequency-dependent propagation constant of the SIIS-loaded SIWs; here, $p$ is fixed to $1 \mathrm{~mm}$ and $g$ is varied from 0.2 to $1.5 \mathrm{~mm}$. The analytical results are calculated using Eq. (1), with $C_{s}$ obtained from Fig. 2(a) (highlighted as stars). From Fig. 2(c), we find that there is a good agreement between the analytical and full-wave simulation results. Since a capacitive SIIS provides an extra phase shift in the transverse direction, a SIIS-loaded SIW exhibits a reduced cut-off frequency for the dominant $\mathrm{TE}_{10}$ mode. Furthermore, the equivalent surface capacitance of SIIS increases upon reducing the gap size of blind vias [see Fig. 2(a)], which may, in turn, reduce the waveguide cut-off frequency. Figure 2(d) is similar to Fig. 2(c), but studying the effect of period on the waveguide dispersion; here, $g$ is fixed to $0.5 \mathrm{~mm}$ and $p$ is varied from 1 to $3.2 \mathrm{~mm}$. Again, we find that the analytical results based on the extracted surface capacitance in Fig. 2(b) (highlighted as stars) agree well with the simulation results. When the pitch distance is reduced, the surface capacitance of SIIS is increased, which further reduces the cut-off frequency. Moreover, the dispersion in the SIIS-loaded SIW also leads to a transition between the slow-wave propagation $(\beta>k)$ and the fast-wave propagation $(\beta<k)$, as can be seen in Figs. 2(c) and 2(d). The simultaneous reduction of cut-off frequency and effective wavelength (associated with the slow-wave prorogation) may enable miniaturization of a waveguide in both transverse and longitudinal dimensions. We should note that loading the bulky negative magnetic metamaterials $(\mu<0)$, such as the three-dimensional (3D) split-ring resonator arrays with permeability described by the Lorentzian mode, could also reduce the physical dimension of the waveguide and cavity [34-39]. However, this technique usually suffers from the large insertion loss (propagation loss) and the intrinsically narrowband response of negative magnetic metamaterials (related to passivity and causality). For example, it was reported in [37] that in the negative permeability band, a high insertion loss (approximately $7 \mathrm{~dB}$ ) is obtained due to the resonant absorption. Compared to those metamaterial-based miniaturization techniques, the proposed SIIS technique not only eases the fabrication complexity and cost, but also allows a measured insertion loss lower than $3 \mathrm{~dB}$, thanks to the nonresonant nature of reactive impedance surfaces.

To validate the theoretical results, we fabricate several prototypes of the SIIS-loaded SIWs and fully characterize their properties. The SIW and SIIS are fabricated using the standard PCB process. The dielectric substrate (F4B) has permittivity $\varepsilon=2.65$ and thickness $t=3 \mathrm{~mm}$. The via holes are drilled in the dielectric substrate to form the two sidewalls of the SIW. The SIIS is fabricated using 


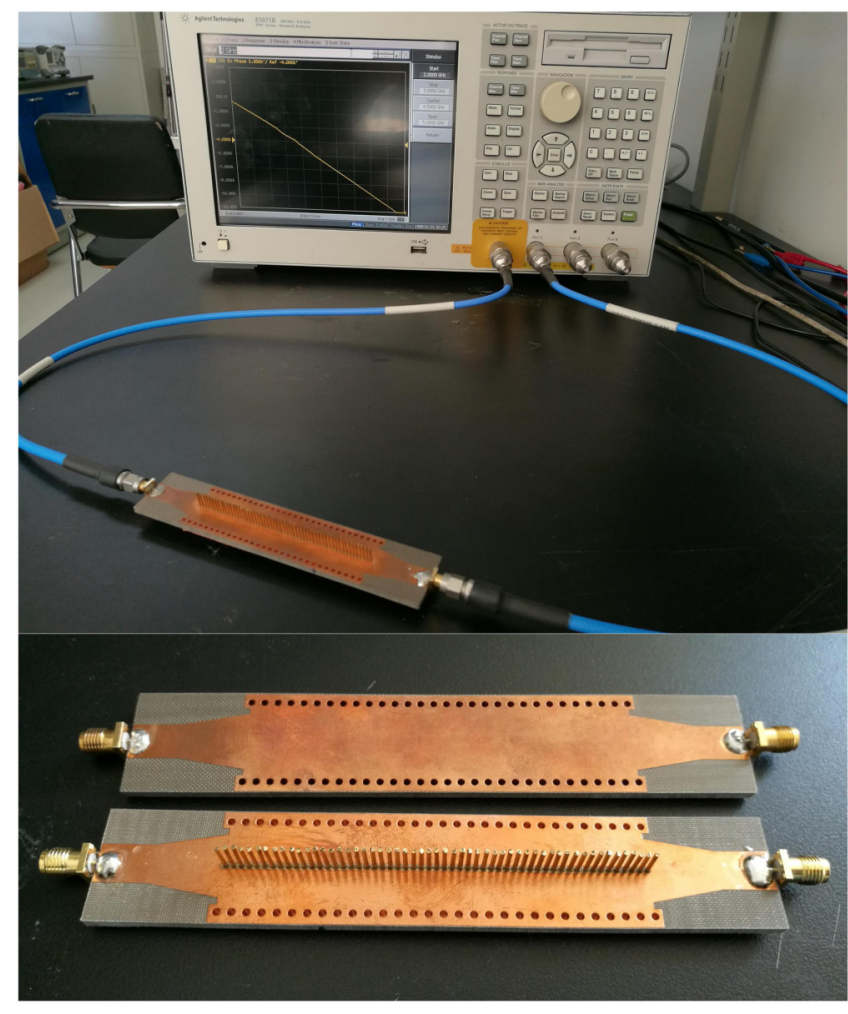

FIG. 3. Photographs of the SIIS-loaded SIW and the standard SIW.

the same mechanical drilling process, with the diameter of via $a=1 \mathrm{~mm}$. Figure 3 shows photographs of the standard SIW and the SIIS-loaded SIW, with length $L=90 \mathrm{~mm}$ and width $W=20 \mathrm{~mm}$. The transmission coefficient $\left(S_{21}\right)$ and the propagation constant are measured using the vector network analyzer (Agilent N5071B).
We first consider a SIIS with a fixed period $(p=1.6 \mathrm{~mm})$ and different gap sizes $(g=0.9$ and $1.5 \mathrm{~mm})$. Figure 4(a) presents the simulated (dashed) and measured (solid) transmission coefficients $\left(S_{21}\right)$ of the standard and SIIS-loaded SIWs in Fig. 3. We find that the experimental and numerical results are in good agreement. Small ripples in the transmission spectrum can be suppressed by further optimizing the microstrip-to-waveguide taper. It is seen from Fig. 4(a) that by loading the capacitive SIIS, the cutoff frequency can be downshifted from $5 \mathrm{GHz}$ (standard SIW) to $4.25 \mathrm{GHz}$ (SIIS-loaded SIW with $g=1.5 \mathrm{~mm}$ ) and $3.75 \mathrm{GHz}$ (SIIS-loaded SIW with $g=0.9 \mathrm{~mm}$ ). As is already known from Fig. 2, the cut-off frequency decreases upon reducing the gap size of blind vias, due to the increased equivalent surface capacitance. For both loaded and unloaded SIWs, a good insertion loss $(<-3 \mathrm{~dB})$ is obtained. Figure 4(b) reports the simulated (dashed) and measured (solid) real part of the propagation constant for the SIWs in Fig. 4(a). Here, the propagation constant of a SIIS-loaded SIW is calculated by solving the transcendental equation [Eq. (1)], with $Z_{s}$ obtained from the analytical formula [Eq. (10)]. From Fig. 4(b), we find that the propagation constant extracted from measurement results agree well with the theoretical results, thus verifying the effectiveness of our analytical model. Figures 4(c) and 4(d) are similar to Figs. 4(a) and 4(b), but studying the effect of period on the cut-off frequency; here, the gap size is fixed $(g=1 \mathrm{~mm})$ and the period is varied $(p=1.6,3.6$, and $4.8 \mathrm{~mm}$ ). In all cases, the simulation and measurement results agree quite well. From Figs. 4(c) and 4(d), it can be observed that when $p$ decreases, the cut-off frequency of the waveguide is downshifted, which is consistent with results presented in Fig. 2. When $p$ is reduced from 4.8 to $1.6 \mathrm{~mm}$, the cut-off frequency of the SIIS-loaded SIW (a)

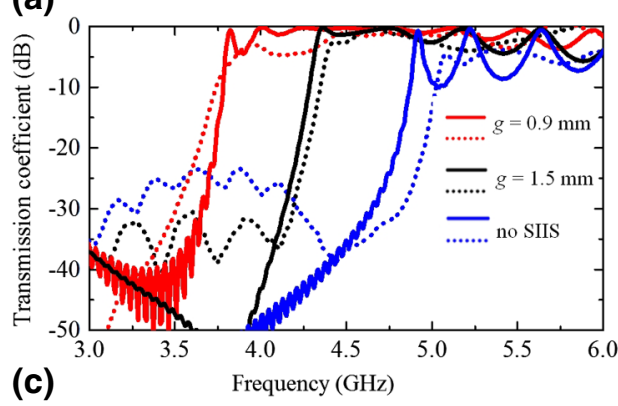

(c)

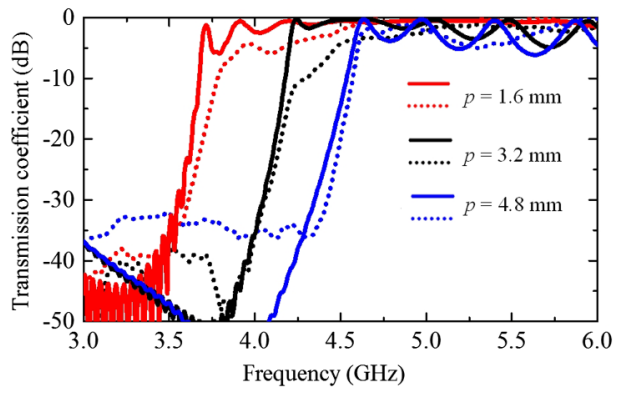

(b)
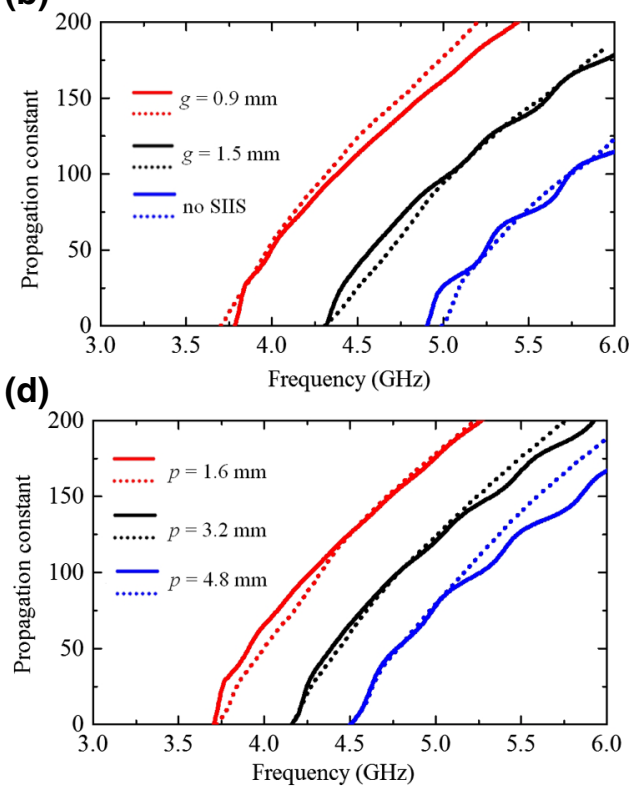

FIG. 4. Experiment demonstration of the SIIS-loaded waveguide with different geometric parameters. Simulation (dashed lines) and measurement (solid lines) results for (a) transmission coefficients $\left(S_{21}\right)$ and (b) real part of propagation constant of the SIIS-loaded SIW in Fig. 3, with different values of $g(p=1.6 \mathrm{~mm})$. (c),(d) are similar to (a),(b), but for the SIISloaded SIW in Fig. 3, with different values of $p(g=1 \mathrm{~mm})$. 
(a)

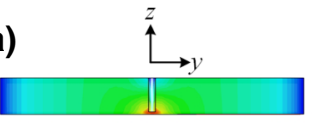

(b)

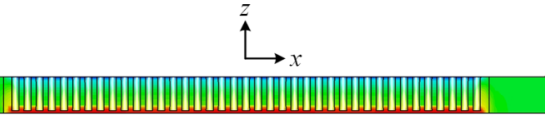

(c)

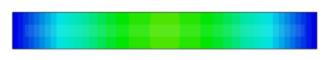

(d)

(e)

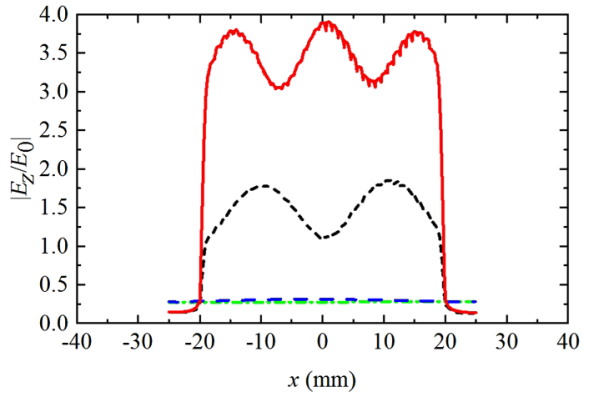

-.--- Rectangular waveguide - - SIW ---- SIIS-loaded SIW $(g=0.5 \mathrm{~mm}) \longrightarrow$ SIIS-loaded SIW $(g=0.2 \mathrm{~mm})$

(f)

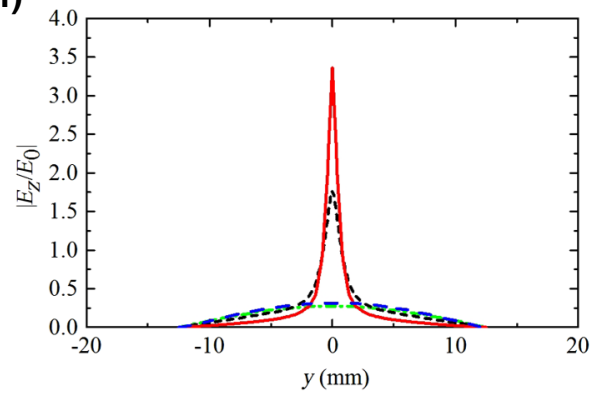

FIG. 5. Distributions of electric field intensity. Distributions of electric field intensity in (a) cross-section view and (b) longitudinal-section view for the SIIS-loaded SIW at $7 \mathrm{GHz}$. (c),(d) are similar to (a),(b), but for the standard SIW. Normalized electric field intensity for the rectangular waveguide, the standard SIW, and the SIIS-loaded SIW (with $g=0.5$ and $0.2 \mathrm{~mm}$ ) along the (e) $x$ axis and (f) $y$ axis. is decreased from 4.5 to $3.7 \mathrm{GHz}$. Tailoring the diameter of blind vias could also vary the surface impedance of SIIS and thus propagation characteristics of the SIISloaded SIW, which is discussed in detail in Supplementary Material [40] as well as the effect of dielectric loss.

The SIW resonators, with a compact size and a high $Q$ factor, have been proposed to make microwave sensors for detecting complex permittivity inside the SIW. For instance, the device can be made by integrating a SIW with a liquid/air-based microfluidic channel [41-43]. In this context, the large and uniform electric field around ENZ frequencies has been proposed to make versatile waveguide-based permittivity sensors $[44,45]$. It can be straightforwardly understood from the perturbation theory, indicating that when a resonant cavity is perturbed by a permittivity change, the shift of resonance frequency is proportional to the local field intensity. Finally, we should note that a miniaturized SIIS-loaded SIW can also be utilized for sensing and concentrating the electric field. Figures 5(a) and 5(b) present the simulation results for distributions of electric field intensity on the cross-section and longitudinal-section planes of the SIIS-loaded SIW [Fig. 2(a)] at 7.0 GHz. Figures 5(c) and 5(d) are similar to Figs. 5(a) and 5(b), but for a standard SIW. Here, the loaded and unloaded SIWs have identical width, length, and thickness, which are $w=25 \mathrm{~mm}, L=40 \mathrm{~mm}$, and $t=3 \mathrm{~mm}$, respectively. From Fig. 5, it is clearly seen that the electric fields can be strongly enhanced and localized around the SIIS, particularly in the gap region. In this sense, under the same permittivity perturbation, the cutoff or ENZ frequency of the SIIS-loaded SIW could be remarkably shifted, well beyond the limit of SIW-based sensors. Figures 5(e) and 5(f), respectively, present the electric field intensity along the longitudinal direction ( $y$ direction) and transverse direction ( $x$ direction) for the rectangular waveguide (green line), the standard SIW (blue line), and the SIIS-loaded SIW (red and black lines); here, all waveguiding structures have identical physical dimensions and operate at the same frequency $(7.0 \mathrm{GHz})$. From Figs. 5(e) and 5(f), we find that the SIIS-loaded SIW exhibits a much stronger local electric field when compared to the standard SIW and the rectangular waveguide. Moreover, the maximum electric field, occurring in the SIIS's gap region, will increase upon reducing the gap between the blindvias array and the ground plane. As can be understood from Eq. (10), reducing the gap size will increase the surface capacitance of SIIS, further squeezing the waveguide dimension and enhancing the local field strength. Such a field concentration effect may be of interest for various waveguide-based microwave sensing, radiation, and nonlinear (e.g., frequency multipliers and mixers) components that are field-intensity dependent.

\section{CONCLUSIONS}

In this article, we have discussed the control of waveguide dispersion of an on-aboard or on-chip waveguide by loading a SIIS, constituted by blind/through-hole vias and multilevel interconnect structures. Different from those metamaterial-loaded miniature waveguides, the proposed SIIS-loaded SIW can not only ease the implementation complexity, but also enable low propagation loss and high power-carrying capacity. We have developed a theoretical framework to model the equivalent surface impedance of a specific SIIS and analyze propagation characteristics of a SIIS-loaded SIW. We have validated the theoretical results with both full-wave simulations and experiments. This SIIS technique shows great potential for engineering 
the dispersion and dimensions of versatile waveguide components. In addition, the SIIS-loaded waveguide exhibits strong local field enhancement, which may be used to boost nonlinearities of active components and to enhance sensitivity of waveguide-based microwave sensors.

\section{ACKNOWLEDGMENTS}

This work is supported by the National Natural Science Foundation of China Grant No. 61771280. P.Y.C. and H.B. would like to thank KAUST Grant No. CRG-2953 for supporting the research reported in this publication.

[1] K. S. Packard, The origin of waveguides: A case of multiple rediscovery, IEEE Trans. Microw. Theory Tech. 32, 961 (1984).

[2] K. Grenier, D. Dubuc, P.-E. Poleni, M. Kumemura, H. Toshiyoshi, T. Fujii, and H. Fujita, Integrated broadband microwave and microfluidic sensor dedicated to bioengineering, IEEE Trans. Microw. Theory Tech. 57, 3246 (2009).

[3] E. Ozbay, Plasmonics: Merging photonics and electronics at nanoscale dimensions, Science 311, 189 (2006).

[4] M. Henry, C. E. Free, B. S. Izqueirdo, J. Batchelor, and P. Young, Millimeter wave substrate integrated waveguide antennas: Design and fabrication analysis, IEEE Trans. Adv. Packag. 32, 93 (2009).

[5] W. Wang, S. S. Zhong, Y. M. Zhang, and X. L. Liang, A broadband slotted ridge waveguide antenna array, IEEE Trans. Antennas Propag. 54, 2416 (2006).

[6] A. E. Atia and A. E. Williams, Narrow-bandpass waveguide filters, IEEE Trans. Microw. Theory Tech. 20, 258 (2003).

[7] D. Deslandes and K. Wu, Single-substrate integration technique of planar circuits and waveguide filters, IEEE Trans. Microw. Theory Tech. 51, 593 (2003).

[8] F. F. He, K. Wu, W. Hong, L. Han, and X. Chen, A low phase-noise VCO using an electronically tunable substrate integrated waveguide resonator, IEEE Trans. Microw. Theory Tech. 58, 3452 (2010).

[9] F. Cheng, X. Lin, K. Song, Y. Jiang, and Y. Fan, Compact diplexer with high isolation using the dual-mode substrate integrated waveguide resonator, IEEE Microw. Wirel. Components Lett. 23, 459 (2013).

[10] B. Liu, W. Hong, Y. Q. Wang, Q. H. Lai, and K. Wu, Half mode substrate integrated waveguide (HMSIW) 3dB coupler, IEEE Microw. Wirel. Components Lett. 17, 22 (2007).

[11] T. Djerafi and K. Wu, Super-compact substrate integrated waveguide cruciform directional coupler, IEEE Microw. Wirel. Components Lett. 17, 757 (2007).

[12] Y. J. Cheng, W. Hong, and K. Wu, Millimetre-wave monopulse antenna incorporating substrate integrated waveguide phase shifter, IET Microw. Antennas Propag. 2, 48 (2008).

[13] K. Sellal, L. Talbi, T. A. Denidni, and J. Lebel, Design and implementation of a substrate integrated waveguide phase shifter, IET Microw. Antennas Propag. 2, 194 (2008).
[14] M. G. Silveirinha and N. Engheta, Tunneling of Electromagnetic Energy Through Subwavelength Channels and Bends Using Epsilon-Near-Zero Materials, Phys. Rev. Lett. 97, 157403 (2006).

[15] M. G. Silveirinha and N. Engheta, Theory of supercoupling, squeezing wave energy, and field confinement in narrow channels and tight bends using $\varepsilon$-near-zero metamaterials, Phys. Rev. B 76, 245109 (2007).

[16] A. Alù, M. G. Silveririnha, and N. Engheta, Transmissionline analysis of $\varepsilon$-near-zero-filled narrow channels, Phys. Rev. E 78, 16604 (2008).

[17] B. Edwards, A. Alù, M. E. Young, M. Silveirinha, and N. Engheta, Experimental Verification of Epsilon-NearZero Metamaterial Coupling and Energy Squeezing Using a Microwave Waveguide, Phys. Rev. Lett. 100, 33903 (2008).

[18] Y. Li and N. Engheta, Supercoupling of surface waves with $\varepsilon$-near-zero metastructures, Phys. Rev. B 90, 201107 (2014).

[19] C. Argyropoulos, P. Y. Chen, G. D'Aguanno, N. Engheta, and A. Alù, Boosting optical nonlinearities in epsilon-nearzero plasmonic channels, Phys. Rev. B 85, 045129 (2012).

[20] A. Capretti, Y. Wang, N. Engheta, and L. D. Negro, Comparative study of second-harmonic generation from epsilon-near-zero indium tin oxide and titanium nitride nanolayers excited in the near-infrared spectral range, ACS Photonics 2, 1 (2015).

[21] M. Z. Alam, L. I. De, and R. W. Boyd, Large optical nonlinearity of indium tin oxide in its epsilon-near-zero region, Science 352, 795 (2016).

[22] A. Alù and N. Engheta, Boosting Molecular Fluorescence With a Plasmonic Nanolauncher, Phys. Rev. Lett. 103, 43902 (2009).

[23] D. Deslandes and K. Wu, Accurate modeling, wave mechanisms, and design considerations of a substrate integrated waveguide, IEEE Trans. Microw. Theory Tech. 54, 2516 (2006).

[24] Y. Cassivi, L. Perregrini, P. Arcioni, M. Bressan, K. Wu, and G. Conciauro, Dispersion characteristics of substrate integrated rectangular waveguide, IEEE Microw. Wirel. Components Lett. 12, 333 (2002).

[25] M. S. Mahani and G. W. Roberts, A mmWave folded substrate integrated waveguide in a $130-\mathrm{nm}$ CMOS process, IEEE Trans. Microw. Theory Tech. 65, 2775 (2017).

[26] H. Xie, L. Belostotski, and M. Okoniewski, A $460 \mathrm{GHz}$ CMOS substrate integrated waveguide slot antenna design, Microw. Opt. Technol. Lett. 58, 347 (2016).

[27] D. M. Pozar, Microwave Engineering, 4nd ed. (John Wiley \& Sons, Inc., New York, 2012).

[28] S. Tretyakov, Analytical Modeling in Applied Electromagnetics (Artech House, Inc., Norwood, MA, 2003).

[29] P. Y. Chen, C. Argyropoulos, G. D’Aguanno, and A. Alù, Enhanced second-harmonic generation by metasurfaceassembled nanomixer and nanocavity, ACS Photonics 2, 1000 (2015).

[30] P. Y. Chen, C. Argyropoulos, and A. Alù, Enhanced nonlinearities using plasmonic nanoantennas, Nanophotonics 1, 221 (2012).

[31] P. Y. Chen and A. Alù, Subwavelength imaging using phase-conjugating nonlinear nanoantenna arrays, Nano Lett. 11, 5514 (2011). 
[32] A. V. Osipov and S. A. Tretyakov, Modern Electromagnetic Scattering Theory with Applications (John Wiley \& Sons, New York, 2017).

[33] CST Microwave Studio, www.cst.com.

[34] N. Engheta, An idea for thin subwavelength cavity resonators using metamaterials with negative permittivity and permeability, IEEE Antennas Wirel. Propag. Lett. 1, 10 (2002).

[35] M. Caiazzo, S. Maci, and N. Engheta, A metamaterial surface for compact cavity resonators, IEEE Antennas Wirel. Propag. Lett. 3, 261 (2004).

[36] A. Alu and N. Engheta, Guided modes in a waveguide filled with a pair of single-negative (SNG), double-negative (DNG), and/or double-positive (DPS) layers, IEEE Trans. Microwave Theory Tech. 52, 199 (2004).

[37] S. Hrabar, J. Bartolic, and Z. Sipus, Waveguide miniaturization using uniaxial negative permeability metamaterial, IEEE Trans. Antennas Propag. 53, 110 (2005).

[38] P. Y. Chen and A. Alù, Dual-mode miniaturized elliptical patch antenna with $\mu$-negative metamaterials, IEEE Antennas Wirel. Propag. Lett. 9, 351 (2010).

[39] P. Y. Chen and A. Alù, Sub-wavelength elliptical patch antenna loaded with $\mu$-negative metamaterials, IEEE Trans. Antennas Propag. 58, 2909 (2010).
[40] See Supplemental Material at http://link.aps.org/supple mental/10.1103/PhysRevApplied.10.064024 for [Effects of Blind Vias Diameter on the Propagation Characteristics].

[41] M. M. Usman and L. Sungjoon, Reusable EGaIn-injected substrate-integrated-waveguide resonator for wireless sensor applications, Sensors 15, 28563 (2015).

[42] E. Silavwe, N. Somjit, and I. D. Robertson, A microfluidicintegrated SIW lab-on-substrate sensor for microliter liquid characterization, IEEE Sens. J. 16, 7628 (2016).

[43] H. Lobato-Morales, A. Corona-Chavez, J. L. OlveraCervantes, R. A. Chavez-Perez, and J. L. Medina-Monroy, Wireless sensing of complex dielectric permittivity of liquids based on the RFID, IEEE Trans. Microw. Theory Tech. 62, 2160 (2014).

[44] A. Alù and N. Engheta, Dielectric sensing in $\varepsilon$-nearzero narrow waveguide channels, Phys. Rev. B 78, 5102 (2008).

[45] H. Lobato-Morales, D. V. B. Murthy, A. Corona-Chavez, J. L. Olvera-Cervantes, J. Martinez-Brito, and L. G. Guerrero-Ojeda, Permittivity measurements at microwave frequencies using epsilon-near-zero (ENZ) tunnel structure, IEEE Trans. Microw. Theory Tech. 59, 1863 (2011). 